\title{
Hipertiroidismo en niños y adolescentes
}

\author{
CLAUDIA GODOY C. ${ }^{1}$, MARCELA ACEVEDO M. ${ }^{1}$, ANTONIO BARRERA N. ${ }^{1}$, \\ ANAHÍ YISMEYIÁN M. ${ }^{1}$, FRANCISCA UGARTE P. ${ }^{1}$
}

1. Unidad Endocrinología Infantil Hospital Exequiel González Cortés. Departamento de Pediatría, Facultad de Medicina, Universidad de Chile, Sede Sur.

\begin{abstract}
Hyperthyroidism in children and adolescents

Background: Hyperthyroidism (HT) prevalence is $0.1 / 100,000$ children and $1 / 100,000$ adolescents and Graves Disease is the most frequent etiology. Objective: To evaluate the clinical presentation, etiology and treatment in hyperthyroid children. Method: Retrospective review of clinical charts of children under 15 years-old, between June 2004 and August 2005. Hyperthyroidism diagnosis was performed with suppressed TSH and increased thyroid hormones levels. Etiological study was done by TRAb, ATPO, ATG, thyroid echotomography and $\mathrm{I}^{131}$ capture. Results: 26 patients were evaluated; $84.6 \%$ females and age at diagnosis was $9.8 \pm 3,5$ years-old (range: $3,8-14,5)$. Goiter was the most frequent clinical sign $(96,2 \%)$, tachicardy and swelling. Etiology: Graves Disease (73\%), Hashitoxicosis (15,3\%) and unknown etiology (11,5\%). Treatment: $88,4 \%$ began with anti-thyroid drugs (DAT): $78 \%$ PTU and 22\% Tiamazol. $62.5 \%$ became euthyroid after 6 months and $79.1 \%$ after 12 months. $31.5 \%$ of GD presented hypothyroidism at $6.3 \pm 4$ months of DAT, requiring LT4 substitution. $\mathrm{I}^{131}$ was applied to 4 children $(16.6 \%) ; 3$ due to hepatic compromise pre or post PTU use and 1 girl for missing treatment, developing a thyrotoxic torment. Thyroidectomy was done in 2 patients $(8.3 \%)$, both with GD; 1 for giant goiter without DAT response at 19 months and 1 for persistant hyperthyroidism after 25 months of DAT. 92\% received $\beta$-blockers (Propanolol) for adrenergic symptoms for $5 \pm 4$ months. Conclusions: Goiter was the most frequent pediatric HT symptom and Graves disease the main etiology. DAT treatment control HT in $76.9 \%$ patients and no adverse reactions with $\mathrm{I}^{131}$ were observed. These results promote DAT treatment as first line in HT management, prefering Tiamazol for its better adherence and less adverse reactions. Radioiodide therapy and thyroidectomy are alternatives if treatment fails in pediatrics HT.

(Key words: hyperthyroidism, children, treatment, goiter, Graves Disease).

Rev Chil Pediatr 2009; 80 (1): 21-29
\end{abstract}

\section{RESUMEN}

El hipertiroidismo (HT) tiene una prevalencia de $0,1 / 100000$ en niños y $1 / 100000$ en adolescentes, siendo la enfermedad de Graves (EG) la etiología más frecuente. Objetivo: Revisar presentación clínica, etiología y manejo de niños con HT. Método: Estudio retrospectivo de fichas clínicas de niños con HT menores de 15

Trabajo recibido el 29 de octubre de 2008, devuelto para corregir el 22 de diciembre de 2008, segunda versión el 07 de enero de 2009, aceptado para publicación el 08 de enero de 2009.

Correspondencia a:

Francisca Ugarte P.

E-mail: ugartepalacios_fca@hotmail.com 
años, evaluados entre Junio/04 y Agosto/05. El diagnóstico de HT se hizo con TSH suprimida y hormonas tiroideas elevadas. El estudio etiológico se realizó en base a anticuerpos TRAb, ATPO, ATG; Ecotomografía tiroidea, y captación de $I^{131}$. Resultados: Se evaluaron 26 pacientes; 84,6\% fueron mujeres. Edad promedio al diagnóstico fue 9,8 $\pm 3,5$ años (rango 3,8 a 14,5). La presentación clínica más frecuente fue bocio (96,2\%), seguidos por taquicardia y sudoración. Etiología: Enfermedad de Graves 73\%, Hashitoxicosis $15,3 \%$ y etiología no precisada 11,5\%. Manejo: 88,4\% inician con drogas antitiroideas (DAT); $78 \%$ PTU y $22 \%$ con Tiamazol. $62,5 \%$ se hizo eutiroideo a los 6 meses y 79,1\% a los 12 meses. El 31,5\% de EG presentó hipotiroidismo a los $6,3 \pm 4$ meses de uso de DAT, requiriendo sustitución con LT4. El I ${ }^{131}$ fue indicado a 4 niños (16,6\%): en 3 casos por compromiso hepático importante pre o post uso de PTU y 1 niña por abandono de tratamiento y reingreso con tormenta tiroidea. Tiroidectomía: se indicó a 2 pacientes $(8,3 \%)$, ambos con EG; uno por bocio gigante, sin respuesta a DAT después de 19 meses de uso y el otro por persistir hipertiroideo después de 25 meses de uso de DAT. El 92\% recibió $\beta$ bloqueador (propanolol) para manejo de los síntomas adrenérgicos, ( $5 \pm 4$ meses). Discusión y conclusiones: El bocio es el síntoma principal en pediatría. La etiología más frecuente es la Enf. de Graves. Las DAT permitieron controlar el HT en $76,9 \%$ de los pacientes, no observamos complicaciones con el uso de $\mathrm{I}^{131}$. Sugerimos el tratamiento inicial con DAT; preferentemente con tiamazol por mayor adherencia y menos complicaciones. La radioyodo terapia y la tiroidectomía son alternativas frente a fracaso de tratamiento en la edad pediátrica.

(Palabras clave: hipertiroidismo, niños, bocio, tratamiento, enfermedad de graves).

Rev Chil Pediatr 2009; 80 (1): 21-29

\section{Introducción}

El hipertiroidismo es un estado de hipermetabolismo causado por el aumento de las hormonas tiroideas. Es un cuadro descrito con más frecuencia en adultos, pues en niños prepúberes tiene una prevalencia reportada de $0,1 / 100000$ y en adolescentes $1 / 100000$, siendo en todas las edades más frecuente en mujeres. La enfermedad autoinmune es la causa más frecuente de patología tiroidea y la enfermedad de Basedow-Graves (EG), producida por la presencia de anticuerpos estimulantes del receptor de TSH, es la etiología principal del hipertiroidismo (95\%). La segunda causa en frecuencia también es autoinmune, la Hashitoxicosis, que corresponde al aumento de secreción de hormonas tiroideas por la actividad inflamatoria inicial de la glándula en respuesta a los anticuerpos antiperoxidasa y antitiroglobulina características de la Tiroiditis linfocitaria crónica.

El cuadro clínico del hipertiroidismo ha sido ampliamente descrito y presenta múltiples manifestaciones siendo lo más representativo el aumento de actividad adrenérgica y la presencia de bocio. El diagnóstico se realiza al constatar una TSH suprimida y/o T3 o T4 en sus fracciones totales y/o libres elevadas. La captación de $\mathrm{I}^{131}$ y el cintigrama $\mathrm{Tc}^{99}$ ayudan en el diagnóstico etiológico. Para el estudio del ori- gen autoinmune de esta enfermedad se miden los anticuerpos anti-receptor de TSH (TRAb), anti tiroperoxidasa (ATPO) y antitiroglobulina (ATG).

Las opciones terapéuticas son uso de drogas antitiroideas (DAT), iodo radioactivo $\left(\mathrm{I}^{131}\right)$ y la tiroidectomía.

Las características de esta patología en la población infantil ha sido poco estudiada ya que es más frecuente en adultos. El objetivo de este trabajo es revisar las características clínicas, etiología y manejo de pacientes con diagnóstico de hipertiroidismo en la población infantil.

\section{Pacientes y Métodos}

Se revisaron en forma retrospectiva las fichas clínicas de pacientes menores de 15 años con diagnóstico de hipertiroidismo, controlados entre el 1 de Julio de 2004 y el 31 de Junio de 2005 en la Unidad de Endocrinología del Hospital de niños Exequiel González Cortés. Se registraron los siguientes datos demográficos: edad al inicio de los síntomas y al momento del diagnóstico, sexo, presencia de otras enfermedades y antecedentes familiares de enfermedad tiroidea en los parientes de primer y segundo grado.

Se definió tirotoxicosis como el estado clíni- 
co resultante de la exposición de los tejidos a cantidades excesivas de hormonas tiroideas ${ }^{1}$ por lo que el diagnóstico se hizo mediante sintomatología clínica sugerente (nerviosismo, sudoración, pérdida de peso, diarrea), exploración física orientadora (bocio, exoftalmos, temblor, taquicardia, HTA) y los resultados de laboratorio. Se realizó una evaluación del estado nutricional al momento del diagnóstico. El diagnóstico de hipertiroidismo se basó en la presencia de TSH suprimida $(<0,27 \mathrm{uUI} / \mathrm{ml})$ y T3-T4 y/o T4L elevadas (Valores normales: T3: 52175 ng/dL; T4: 6,1-14,4 ug/dL; T4 Libre: 0,8-2,0 $\mathrm{ng} / \mathrm{dL})^{1}$

El estudio etiológico incluyó medición de anticuerpos estimuladores del receptor de TSH (Thyroid Stimulating Receptor Antibodies (TRAb)) Valor Normal < 11\% de inhibición; Anti Tiroperoxidasa (ATPO) y Anti Tiroglobulina (ATG), captación de $\mathrm{I}^{131}$ y ecotomografía tiroidea.

La enfermedad de Graves se definió por niveles de TRAbs aumentados y/o captación $I^{131}$ elevada en pacientes cuya ecotomografía tiroidea descartó nódulos.

Se describe el tipo de tratamiento utilizado como drogas antitiroideas (Propiltiouracilo o Tiamazol), I ${ }^{131}$, o tiroidectomía. La respuesta al tratamiento fue analizada con la evaluación clínica y medición seriada de T3, T4 y/o T4 libre y TSH. Se reportaron las complicaciones derivadas de cada tratamiento. Se calculó el tiempo de seguimiento de los pacientes desde el momento del diagnóstico hasta la fecha del estudio.

Los resultados se expresan como frecuencia observada y como promedios \pm DS y rango, según corresponda.

\section{Resultados}

Se revisaron las fichas clínicas de 29 pacientes de las que se excluyeron 3 por corresponder a hijos de madres con Enfermedad de Graves, que presentaron un hipertiroidismo neonatal transitorio. Veintidós del total de 26 $(84,6 \%)$ de los pacientes fueron de sexo femenino y 3 de los pacientes son portadores del Síndrome de Down (11\%).
Los síntomas se iniciaron a los $8,9 \pm 4,1$ años de edad (rango 0,16-14,3) y el diagnóstico se hizo a los 9,8 $\pm 3,5$ años (rango 3,8 a 14,5). La duración de los síntomas antes del inicio del tratamiento fue de 6,6 $\pm 10,6$ meses (mediana 3 meses, rango 1 a 48 meses). Un paciente varón inició los síntomas a los 2 meses de vida, destacando mal incremento de peso, talla sobre el percentil 95 para la edad, hiperactividad moderada y diarrea 2 a 3 veces al mes; fue derivado a los 4 años de edad al constatar bocio evidente y exoftalmo (al ingreso tenía: TSH 0,001 uU/ml, T4 28,2 ug/dl (VR: 6,1-14,4) y T3 356 ng/dL (VR: 52-175), TRAb + 63\% (VR $<11 \%)$.

El antecedente de algún familiar de primer o segundo grado con enfermedad tiroidea ocurrió en $42 \%$ de los casos, no siendo posible objetivar que patología, pues la mayoría sólo refirió el diagnóstico de bocio.

La distribución por edad y sexo se muestra en la figura 1, destacando mayor frecuencia en mujeres $(84,6 \%)$ y en el grupo de 10 a 14,9 años (57,6\%). La distribución según el tipo de síntomas se muestra en la tabla 1 , siendo los síntomas más frecuentes bocio $(96,2 \%)$, sudoración $(80,7 \%)$ y taquicardia $(77 \%)$.

La evaluación de laboratorio inicial mostró que todos los pacientes tenían TSH suprimida, $(\mathrm{x}=0,05 \pm 0,07 \mathrm{DS})$. Los valores de T4, T4 libre y/o T3 estaban elevados en todos excepto 3 pacientes: 2 con EG y 1 con Hashitoxicoxis en que estaban en el rango máximo de lo normal. Los valores promedios fueron T4: 17,4 \pm 7,2 ug/dL; T3: $410 \pm 220,6 \mathrm{ng} / \mathrm{dL}$; T4L: 3,0 $\pm 1,5$ $\mathrm{ng} / \mathrm{dL}$. En la tabla 2 se muestran los valores de estos y de los otros exámenes de laboratorio.

Se realizó ecotomografía tiroidea en 23 casos, 19 mostraron bocio difuso, 3 bocio multinodular y 1 fue normal. Los 3 pacientes con bocio multinodular tenían anticuerpos TRAb $(+)$, por lo que se interpretó como EG y los nódulos desaparecieron en el transcurso del seguimiento en todos ellos. La ecografía de tiroides fue normal en un paciente con Hashitoxicosis.

La causa más frecuente de HT fue la enfermedad de Graves ( $73 \%$ de los casos); en el $15,3 \%(4 / 26)$ la etiología correspondió a una Hashitoxicosis y en el $11,5 \%$ de los casos (3/ 26) no se logró concluir etiología (figura 2). 


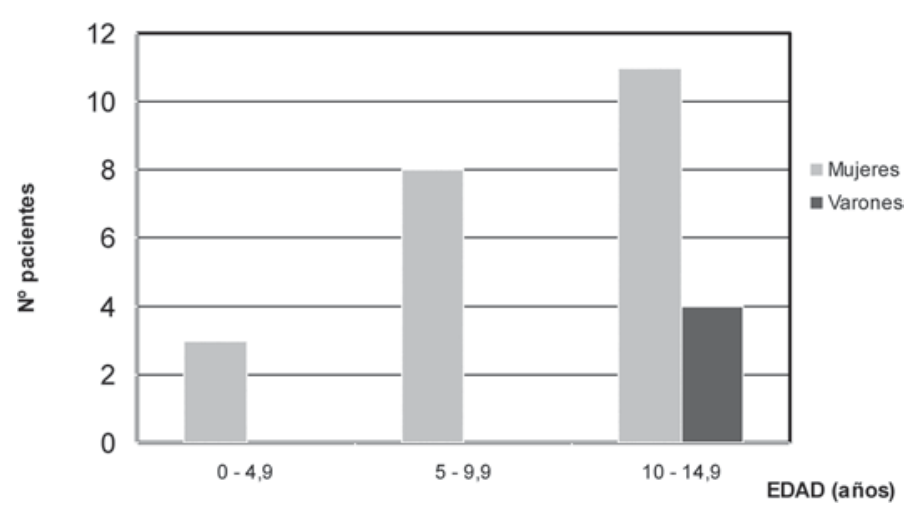

Figura 1. Distribución por grupo etario y sexo, pacientes pediátricos con hipertiroidismo.
Tabla 1. Frecuencia de signos y síntomas, pacientes pediátricos con hipertiroidismo

\begin{tabular}{llr}
\hline Síntomas y signos & \% & (n) \\
\hline Bocio & 96,2 & $(25)$ \\
Sudoración & 80,7 & $(21)$ \\
Taquicardia & 77 & $(20)$ \\
Intolerancia al calor & 61,5 & $(16)$ \\
Palpitaciones & 57,6 & $(15)$ \\
Exoftalmo & 57,6 & $(15)$ \\
Hiperactividad & 57,6 & $(15)$ \\
Temblor & 53,8 & $(14)$ \\
Baja de peso & 50 & $(13)$ \\
Diarrea & 19,2 & $(5)$ \\
HTA & 11,5 & $(3)$ \\
\hline
\end{tabular}

Tabla 2. Exámenes de Laboratorio al diagnóstico, pacientes pediátricos con hipertiroidismo

\begin{tabular}{lccc}
\hline & & \multicolumn{1}{c}{$\mathbf{x} \pm \mathbf{d s}$} & \multicolumn{1}{c}{ Valor Normal } \\
\hline TSH & $(\mathrm{uUl} / \mathrm{ml})$ & $0,05 \pm 0,07$ & $0,27-4,22$ \\
T4 & $(\mathrm{ug} / \mathrm{dL})$ & $17,4 \pm 7,2$ & $6,1-14,4$ \\
T4L & $(\mathrm{ng} / \mathrm{dL})$ & $3,0 \pm 1,5$ & $0,8-2,0$ \\
T3 & $(\mathrm{ng} / \mathrm{dL})$ & $410 \pm 220$ & $52-175$ \\
TRAb $+(\%) \mathrm{n}=17$ & $60,7 \pm 2,1$ & $<11 \%$ \\
ATPO & $(\mathrm{Ul} / \mathrm{ml})$ & 371 & $<12$ \\
ATG & $(\mathrm{Ul} / \mathrm{ml})$ & 564 & $<34$ \\
\hline
\end{tabular}

* thyroid receptor antibody; ${ }^{* *}$ anticuerpos antitiroperoxidasa; ${ }^{* * *}$ anticuerpos antitiroglobulina.

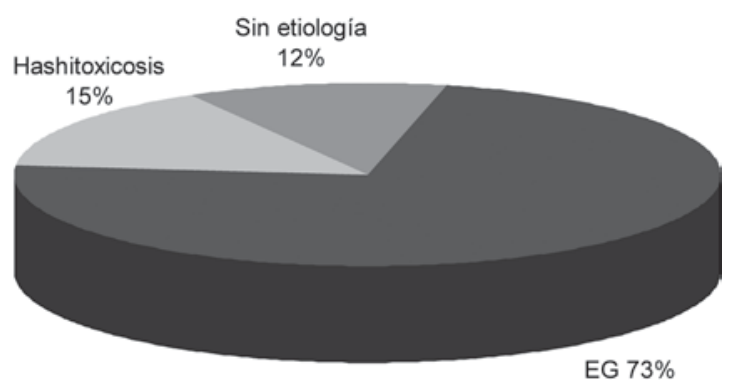

Figura 2. Etiología del hipertiroidismo en pacientes pediátricos.

Se hizo el diagnóstico de EG a 19 pacientes; en 17 de ellos por TRAb positivos (TRAb promedio: $60,7 \pm 2,1 \%$ al inicio, con VR $<11 \%$ ). En 3 de éstos pacientes además tenían captación con $\mathrm{I}^{131}$ elevada; sólo en 2 casos en que no realizaron anticuerpos TRAb por motivos económicos, el diagnóstico de EG se realizó con captación tiroidea de $\mathrm{I}^{131}$ elevada (85 y $99 \%$ respectivamente), y la presencia en ambos de bocio difuso descrito en la ecotomografía tiroidea; uno de los pacientes además tenía exoftalmo bilateral. Trece pacientes con EG (68\%) tenían ATPO (+) (81 a $1076 \mathrm{UI} / \mathrm{ml})$ o ATG (+) (50$560 \mathrm{UI} / \mathrm{ml})$.

Se diagnosticó Hashitoxicosis a 4 pacientes, todas de sexo femenino quienes debutaron con clínica de tirotoxicosis, TSH suprimido, hormonas tiroideas elevadas y bocio difuso. 2 de ellas con TRAb $<11 \%$ y ATPO elevados (460 y $>1000 \mathrm{UI} / \mathrm{ml}$ ). Ambas recibieron tratamiento con Propiltiuracilo, haciéndose hipotiroideas a los 4 y 12 meses respectivamente, comportándose como Tiroiditis linfocitaria crónica posterior a la suspensión del tratamiento con PTU y requiriendo Levotiroxina. La $3^{\mathrm{a}}$ paciente debutó con TSH 0,09 uU/ml, T4 15,9 ug/dl, ATG $2856 \mathrm{UI} / \mathrm{ml}$, no pudo realizarse TRAb. Se manejó con $\beta$ bloqueadores por 2 meses haciéndo- 
se posteriormente hipotiroidea. La última paciente presentó al inicio TSH $0,06 \mathrm{uU} / \mathrm{ml}$, T4L 2,2 ng/dl, T3 2,34 ng/ml, TRAb 18\%, ATPO $164 \mathrm{UI} / \mathrm{ml}$, ATG $186 \mathrm{UI} / \mathrm{ml}$, captación de I31 $12 \%$; se manejó 3 meses con $\beta$ bloqueadores, a los 12 meses de seguimiento tenía TSH 3,88 $\mathrm{uU} / \mathrm{ml}$, T4L $0,8 \mathrm{ng} / \mathrm{dl}$ y clínicamente estaba eutiroidea aún.

En 3 pacientes no se ha podido establecer la etiología exacta del hipertiroidismo, pues los pacientes han sido inconstantes en sus controles o no se han realizado los exámenes necesarios. Los 3 pacientes tienen bocio difuso, por lo que no corresponden a nódulos hiperfuncionantes. Se manejaron con DAT, con respuesta clínica y de laboratorio adecuada, impresionan corresponder a EG.

Respecto del tratamiento, para el manejo de los síntomas adrenérgicos se usaron $\beta$ bloqueadores en $92 \%$ de los niños, por 3,5 \pm 2 meses en promedio. Sólo 2 niñas, con diagnóstico de Hashitoxicosis, recibieron tratamiento exclusivo con $\beta$ bloqueadores. Los 24 restantes se manejaron con DAT, $\mathrm{I}^{131}$ o tiroidectomía. Las drogas antitiroideas fueron el tratamiento de primera línea en 23 pacientes, usaron PTU $78 \%$ (18 pacientes) y Tiamazol (Thyrozol $\left.{ }^{\circledR}\right)$ $22 \%$ (5 pacientes). $62,5 \%$ de los pacientes se hicieron eutiroideo a los 6 meses y 79,1\% a los 12 meses. El 31,5\% (6/19) de EG presentó hipotiroidismo a los 6,3 \pm 4 meses de uso de DAT, requiriendo sustitución con LT4.

Sólo 3 pacientes habían suspendido las DAT al momento del estudio como fin del tratamiento, con tiempos de tratamientos de 12,15 y 19 meses respectivamente. Ninguno había recurrido a los 12 meses de suspensión.

El I ${ }^{131}$ fue indicado a 4 niños $(16,6 \%)$ : en un caso fue el tratamiento de elección desde el inicio por tener el paciente compromiso hepático importante previo al inicio de la terapia. 2 pacientes lo recibieron por presentar elevación significativa de transaminasas, y 1 niña que abandona tratamiento por problemas familiares y reingresa con clínica de tormenta tiroidea.

Se indicó tiroidectomía en 2 pacientes $(8,3 \%)$, ambos con EG; uno por bocio gigante, sin respuesta a DAT después de 19 meses de uso y el otro por persistir hipertiroideo después de 25 meses de uso de DAT.
En cuanto a complicaciones de tratamiento, $15 \%$ de los casos tratados con drogas antitiroideas presentaron alza significativa de las transaminasas, que requirió suspensión de la droga y optar por terapia alternativa. No se registraron alteraciones en el hemograma. No hubo complicaciones derivadas de $\mathrm{I}^{131} \mathrm{ni}$ de la tiroidectomía a la fecha del estudio. El tiempo de seguimiento de los pacientes fue de 1,9 $\pm 1,4$ años hasta el momento del estudio.

\section{Discusión}

Las hormonas tiroideas juegan un rol crucial en el crecimiento y desarrollo del niño, participando en la mielinización del SNC, en el crecimiento, en la pubertad y en el metabolismo de los distintos órganos por lo que la disfunción tiroidea podría causar daños irreversibles durante la infancia ${ }^{2}$.

La tirotoxicosis es un desorden raro en la infancia que resulta del exceso de hormonas tiroideas circulantes y hace referencia a sus manifestaciones clínicas, fisiológicas y bioquímicas; cuando esta es causada por una enfermedad de la glándula tiroides el cuadro se denominada Hipertiroidismo. ${ }^{3,4}$ Nuestro estudio describe las características clínicas, epidemiológicas, de laboratorio y los resultados del tratamiento de 26 pacientes controlados en el plazo de 1 año en la Unidad de Endocrinología del Hospital Exequiel González Cortés y que generan al 1,19\% de las consultas anuales en nuestra unidad. Dado que es una muestra no representativa de la población por ser nuestro centro de derivación de esta patología, no es posible obtener datos de prevalencia ni incidencia poblacionales; la literatura describe una incidencia de este trastorno de 0,1 por 100000 en niños prepuberales y de 3 por 100000 en adolescentes $^{3,5}$. Puede desarrollarse en la infancia pero es raro en los menores de 5 años, diagnosticándose en la etapa puberal en la mayoría de los casos, con un peak entre los 11-15 años. Una publicación pediátrica nacional reciente, describe que $55,2 \%$ tenía 10 años o menos $^{6}$, sin embargo, en nuestros pacientes la edad de presentación más frecuente fue entre los 10 y 15 años y sólo en este rango etario hubo pacientes 
de sexo masculino. Esta distribución por sexo concuerda con lo descrito en la literatura, en que la relación mujeres:hombres es entre 5:1 y $8: 1^{7}$. Es más prevalente en pacientes con síndrome de Down ${ }^{8}$ y deleciones del cromosoma 22q; en nuestro grupo hubo 3 pacientes con síndrome de Down y en el de Martínez y cols $2 / 38^{6}$.

Los síntomas son variados, y de presentación larvada, lo que en general demora el diagnóstico en promedio unos 8 meses en los prepúberes y 5 meses en los púberes 5 . En nuestra casuística la demora del diagnóstico fue de 6,6 meses, destacando un lactante que inició sus síntomas a los 2 meses de edad caracterizados por mal incremento de peso, talla alta e hiperactividad, y realizándose el diagnóstico a los 4 años de edad al aparecer bocio. De hecho el signo más frecuente de observar es bocio, que está descrito en casi el $100 \%$ de los niños con HT, presentándose en nuestro grupo en el $92 \%$ de los niños. Se describen también signos como hiperquinesia o hiperactividad que a veces se manifiesta como trastorno de aprendizaje o falta de atención ${ }^{9}$; aparece irritabilidad, insomnio, fatiga, debilidad muscular, intolerancia al calor, diarrea y otros signos de actividad adrenérgica como sudoración, taquicardia, palpitaciones y aumento de la presión arterial ${ }^{10}$. También se describe pérdida de peso con apetito conservado o incluso aumentado. Existe aumento de velocidad de crecimiento con adelanto de la edad ósea especialmente en prepúberes ${ }^{7}$. Todos estos síntomas se presentaron en distintos grados en nuestros pacientes, como consulta espontánea o en una anamnesis dirigida.

Si el HT se inicia antes de la pubertad, puede retrasar el inicio puberal, y si se presenta en niñas posmenárquicas puede generar alteraciones menstruales como polimenorrea y menos frecuentemente oligo amenorrea. En los varones puede aparecer ginecomastia. A diferencia de los adultos, en los niños son infrecuentes el mixedema pretibial o la tormenta tiroidea: sólo una de nuestras pacientes la presentó, posterior al abandono total del tratamiento ${ }^{11}$.

La oftalmopatía es un proceso autoinmune fuertemente relacionado con EG; no siempre aparece al mismo tiempo que el HT y no se relaciona con la severidad bioquímica de éste. Se ve en el 25-63\% de los pacientes, siendo la oftalmopatía severa infrecuente en niños ${ }^{12}$. El $57 \%$ de nuestros pacientes presentaron algún grado de oftalmopatía, que disminuyó en la medida que fueron tratados del HT, levemente superior a la otra publicación nacional ${ }^{6}$. La literatura aconseja que todos los pacientes con síntomas oculares significativos como quemosis, proptosis o alteración de la motilidad ocular sean evaluados por un oftalmólogo con experiencia en esta patología ${ }^{5}$.

Para el diagnóstico de HT se requiere niveles de hormonas tiroideas elevadas y TSH suprimida. En etapas tempranas de la enfermedad la TSH podría estar suprimida pero con hormonas tiroideas normales ${ }^{13}$. El cintigrama tiroideo y la captación de yodo no son necesarios para el estudio del HT en forma rutinaria, pero son de utilidad cuando el diagnóstico etiológico es dudoso como ocurre en el síndrome de Mc-Albrigth o algunos casos de Hashitoxicosis para diferenciar de EG. La ecografía tiroidea es útil para diferenciar un bocio nodular tóxico de una $\mathrm{EG}^{3,6}$. En nuestro grupo se realizó cintigrama tiroideo en 4 pacientes y captación de Yodo en 7 pacientes con la principal finalidad de diferenciar EG de Hashitoxicosis. Se realizó ecografía en 24 pacientes para descartar nódulos tiroideos que fueran hiperfuncionantes.

La mayoría de los pacientes pediátricos tienen como causa del HT un desorden autoinmune y es más frecuente en niños con otros desórdenes autoinmunes, como la Diabetes Mellitus 1, Enfermedad de Adisson y Enfermedad Celíaca entre otros ${ }^{5}$, asociación que no fue detectada en nuestro grupo. La causa más comúnmente descrita de HT es la enfermedad de Graves $(95 \%)^{14}$, en nuestro estudio correspondió sólo al $73 \%$ de los casos; esto pudiera explicarse porque en 3 pacientes no se pudo precisar la etiología del HT. Otras causas son la tiroiditis linfocitaria crónica que en su fase inicial produce liberación de hormonas tiroideas por destrucción celular (Hashitoxicosis) ${ }^{15}$, que correspondió en nuestros niños al $15,3 \%$ de las etiologías; se ha descrito también HT inducido por yodo, Síndrome de McCune-Albright, cáncer de tiroides, hipersecreción de TSH e inges- 
tión de hormonas tiroideas ${ }^{16}$. El HT neonatal puede ocurrir en hijos de madre con EG, el cual es transitorio o ser secundario a HT congénito no autoinmune. En nuestro estudio se excluyeron los 3 casos de HT transitorio hijos de madres con EG. En 1 paciente de la serie las manifestaciones clínicas se iniciaron en los primeros meses de vida, y se comprobó un origen autoinmune no hereditario ni transitorio, sino adquirido y persistente (EG).

La EG es un desorden autoinmune caracterizado por bocio difuso, hipertiroidismo y oftalmopatía $^{14}$ y es causado por la acción de anticuerpos IgG anti receptor de TSH en las células tiroideas provocando un efecto estimulante y una sobreproducción y liberación de hormonas tiroideas. Aproximadamente el $75 \%$ de los pacientes con enfermedad de Graves tienen además anticuerpos antiperoxidasa (ATPO) y 25 a 55\% antitiroglobulina (ATG). En nuestros pacientes esta asociación se describió en un $68 \%$ de los niños. En estos casos existe infiltración tiroidea con linfocitos $\mathrm{T}$ y $\mathrm{B}$ y células plasmáticas que es mucho menos extensa que en la enfermedad de Hashimoto ${ }^{17}$. La susceptibilidad a EG está determinada por una mezcla de factores genéticos, ambientales y endógenos. Los mecanismos involucrados son desconocidos y no se ha descrito un gen único responsable, aunque alrededor del $80 \%$ de la susceptibilidad a enfermedad tiroidea autoinmune está determinada por factores genéticos. Dos loci están inequívocamente asociados con el desarrollo de EG, llamados MHC (cromosoma 6p21) y CTLA4 (2q33). Hay también una asociación bien establecida con ciertos alelos HLA, que varían entre los distintos grupos raciales ${ }^{18}$.

No se conoce ningún tratamiento que corrija el desorden autoinmune de la EG dado que todas las terapias se orientan disminuir la hiperfunción de la glándula tiroidea. Las drogas antitiroideas siguen siendo de primera elección en niños y adolescentes ${ }^{19}$; son moléculas simples conocidas como tionamidas e incluyen el Propiltiuracilo (PTU), Metimazol (MTZ) y Carbimazol (CBZ), un análogo de $\mathrm{Metimazol}^{20}$. No se recomiendan como tratamiento en los bocios multinodulares ni en nódulo solitario autónomo porque en estos casos la remisión raramente ocurre ${ }^{19}$.
Las DAT inhiben la síntesis de hormonas tiroideas interfiriendo la oxidación y unión de yodo a la célula tiroidea. Poseen también efectos inmunosupresores importantes, disminuyendo las concentraciones de TRAb y de IL-2 e IL-6. Hay también evidencias de que las DAT inducen apoptosis de linfocitos intratiroideos ${ }^{15}$. La elección de la DAT varía de un país a otro. En USA prefieren el uso de PTU y MTZ; en Europa y Asia usan MTZ y en Unión Soviética utilizan CBZ.

En Chile están disponibles el PTU y el Tiamazol (metimazol). Una diferencia importante entre ambos es que el primero es de vida media más corta $^{21}$ requiriendo su uso 2 a 3 veces al día y el $2^{\circ}$ sólo una vez al día, lo que mejora la adherencia al tratamiento. Las dosis descritas en niños son PTU 5-7 mg/K/día y MTZ 0,3-0,5 mg/K/día, no requiriendo ajustes en pacientes con insuficiencia renal ni hepática. Dosis más altas de DAT producen un control más rápido de las hormonas tiroideas, más no influyen en la tasa de remisión a largo plazo. El uso concomitante de Levotiroxina con DAT ha sido descrito como beneficioso para aumentar la tasa de remisión por Hashizume en $1991^{22}$, pero no se ha podido replicar estos resultados posteriormente.

Los estudios demuestran que en términos de eficacia ambas drogas serían equivalentes ${ }^{23}$. La tasa de remisión con DAT, que se define como permanecer bioquímicamente eutiroideo 1 año después de la suspensión del tratamiento, varía de 15 a $60 \%$ dependiendo de forma inversa a los niveles de $\mathrm{TRAb}^{24}$, al tamaño del bocio (a menor tamaño, mayor remisión) y a la severidad de la enfermedad al inicio de la terapia ${ }^{23}$. Dado el plazo limitado de seguimiento de nuestros pacientes no es posible establecer en ellos el porcentaje de remisión del HT. El tiempo de tratamiento recomendado en adultos es de 12 a 18 meses, dado que tiempos más prolongados no logran tasas mayores de remisión ${ }^{19,23}$. En niños los plazos de tratamiento son mayores y menos consensuados ${ }^{19}$.

Con ambas DAT se han descrito algunos efectos adversos menores en 1-5\% como artralgias, urticaria y trastornos gastrointestinales, que no obligarían a suspender la terapia ${ }^{23}$. Efectos mayores como agranulocitosis $(0,37 \%$ am- 
bas drogas), ocurre en los primeros 3 meses de tratamiento y predomina en pacientes de mayor edad. Otros efectos adversos mayores vistos casi exclusivamente con el uso de PTU son la hepatotoxicidad que se ve en un 0,1 a $0,2 \%$ (destacando que hasta un $28 \%$ presenta elevación leve de las transaminasas que no configuran el cuadro de hepatitis) y menos frecuentemente vasculitis. Estas últimas alteraciones requieren suspensión de la DAT y evaluar terapias alternativas ${ }^{19,25}$. Con el MTZ las tasas de complicaciones descritas son dosis dependientes, siendo casi nulas con dosis menores de 10 mg/día, no así con el uso de PTU. En los pacientes de nuestro estudio la única complicación que se presentó fue elevación significativa de las transaminasas en 2 pacientes que usaban PTU, por lo que se discontinuó el tratamiento.

El tratamiento sintomático es importante cuando la actividad adrenérgica es intensa, se utilizan b bloqueadores (Propanolol o Atenolol) durante el tiempo que duren los síntomas ${ }^{5}$.

La tiroidectomía es el tratamiento de $2^{\circ}$ línea cuando falla el tratamiento con DAT en Europa, sin embargo, en USA es el $\mathrm{I}^{131}$ el tratamiento de elección incluso de primera línea. Las indicaciones actuales de tiroidectomía son EG con bocio muy grande que de síntomas por compresión, bocios multinodulares o nódulos autónomos de gran tamaño ${ }^{4}$, o en presencia de oftalmopatía severa.

La tiroidectomía total (TT) por sobre la subtotal es la actualmente recomendada en la $\mathrm{EG}$, porque diminuye las tasas de recurrencia, tiene un posible efecto beneficioso sobre oftalmopatía y disminuye la posibilidad de cáncer tiroideo en el remanente ${ }^{4}$. En centros de referencia se logra hasta un $98 \%$ de remisión del HT con la TT. Con cirugía subtotal se desarrolla hipotiroidismo en $60 \%$ de los pacientes pero aumenta la recurrencia de hipertiroidismo en un $10-15 \%$. Las complicaciones propias de la cirugía se reducen en manos de un cirujano experto, sin embargo, se describe hipoparatiroidismo permanente en 1,75\%, lesiones del nervio laringeo recurrente $(2 \%)$ y queloide de la cicatriz en un $12,3 \%{ }^{26}$. La mortalidad es de 1/1 000 tiroidectomías realizadas ${ }^{3}$.

La terapia con $\mathrm{I}^{131}$ se realiza desde hace 60 años, y en niños desde la década del 60. El I I31 dado en forma oral emite radiaciones $\beta$ y $\gamma$ que destruyen las células foliculares con inflamación, necrosis, infiltración linfocitaria y fibrosis posterior de la glándula ${ }^{15}$. Su uso está indicado cuando hay falla del tratamiento con DAT o recurrencia de HTR en tiroidectomía subtotal.

La decisión de la terapia de elección al fracasar el tratamiento de DAT en niños se basa en la experiencia de cada centro con Tiroidectomía o ${ }^{131}$. Sin embargo, este último ha sido cada vez más utilizado en este grupo etáreo, dado la aparición de diversos estudios que demuestran la eficacia de este tratamiento. Read en $2004{ }^{27}$ publicó una serie de 116 pacientes con HT cuya edad promedio al inicio del tratamiento con $\mathrm{I}^{131}$ fue de 15 años (3,58 a 19 años) y los evaluaron hasta 36,2 años después. Al usar dosis < de 5,3 mCi tenían una recidiva de $36 \%$, que disminuyó significativamente al usar dosis mayores de $6 \mathrm{mCi}$. Reportó 32\% de hipotiroidismo el primer año post terapia, que aumentó 2,9\% cada año siguiente, por lo que a los 36 años de seguimiento aparece en el $97,9 \%$ de los casos. Los embarazos e hijos de los pacientes, presentaron 3,2\% de anomalías congénitas, tasa similar a la población general (3-4\%), 2 pacientes presentaron neoplasia, una de colon y otra de mama, no hubo leucemias ni cáncer de tiroides. Los autores concluyen que el $\mathrm{I}^{131}$ es un tratamiento seguro y efectivo para el HT por EG, pero debe usarse en dosis altas para asegurar la ablación de la glándula y minimizar el riesgo de cáncer de tiroides que eventualmente podría aparecer en el tejido remanente. En relación a esto, estudios de 10-20 años de seguimiento a pacientes con EG, describen que el riesgo de neoplasia con el uso de DAT es 5 veces mayor si se compara con ${ }^{131}$ y 8 veces si se compara con el tratamiento quirúrgico ${ }^{25}$.

En nuestro estudio, 6 niños requirieron tratamiento de segunda línea: 4 recibieron $\mathrm{I}^{131}$, tres por compromiso hepático previo o post uso de DAT y uno por ser un caso social requirió tiroidectomía total, a ambos por falla de respuesta a DAT, y uno además por presentar bocio gigante.

En conclusión podemos decir que el HT en niños y adolescentes es infrecuente, sin embargo, la gran variedad de signos clínicos que 
presenta debe alertar a los pediatras para evitar el retraso en su diagnóstico y manejo, que puede dejar secuelas importantes en el paciente, en especial a edades menores en que se presenta de forma más severa y la remisión es más lenta.

La causa más frecuente del HT es la enfermedad autoinmune (EG) y el tratamiento de elección son las DAT. En caso de fallar estas, se debe optar por Tiroidectomía total o $\mathrm{I}^{131}$ según la experiencia del centro con cada una y las características del paciente y su enfermedad en particular, no existiendo consenso claro en la actualidad acerca de la superioridad de una sobre la otra.

\section{Referencias}

1.- Read C Jr, Tansey M, Menda Y: A 36-year retrospective análisis of efficay and safety of radioactive iodine in treating young Graves patients. J Clin Endocrinol Metab 2001; 14: 229-43.

2.- Bettendorf $M$ : Thyroid disorders in children from birth to adolescence. 2002 Eur J Nucl Med 29: 439-45.

3.- Jhonstone HC, Dharmaraj P, Cheetham TD: The evaluation and management of thyrotoxicosis. Current Paediatrics 2004; 14: 430-7.

4.- Abós MD, Banzo J, Razola P, Prats E, Ubieto MA: Tratamiento del hipertiroidismo con I131. Rev Esp Med Nuclear 2003; 22: 262-76.

5.- Birrel G, Cheetman T: Juvenil Tirotoxicosis, Can we do better? Arch Dis Child 2004; 89: 745-50.

6.- Zanolli M, Araya A, Cattani A, Orellana P, Martinez A: Enfermedad de Basedow Graves en pacientes pediátricos. Rev Chil Pediatr 2008; 79: 26-35.

7.- Kraiem Z, Newfield RS: Grave's disease in childhood. J Ped Endocrinol Metab 2001; 14: 229-43.

8.- Sanz J. Rev Méd Chil 1999; 127: 967-9.

9.- Webster J, Taback S, Sellers E, Dean H: Grave's disease in children. JAMC 2003; 169: 104-5.

10.- Osman F, Franklyn J, Daykin J, Chwdhary S, Holder $R$, Gammage $M$ : Heart rate variability and turbulence in hiperthyroidism before, during and after treatment Am J Cardiol 2004; 94: 465-69.

11.- Iorcansky S, Herzovich V: Hipertiroidismo infantojuvenil. $2^{\circ}$ edición Texto Endocrinología del niño y el adolescente. Ed. Beas F. Ed. Mediterráneo. Santiago, Chile 2002; 151-57.
12.- Prabhakar BS, Hahn RS, Smith TJ: Current Perspective on Pathogenesis of Graves disease and ophthalmopaty. Endocrine Review 2003; 24: 802-35.

13.- Rubio O, Muñoz M, Pozo I, Argente I: Enfermedad de Graves: estado actual y revisión de 20 casos. Ann Pediatr 2004; 64:131-6

14.- Rivkees S, Sklar Ch, Freemark M: Clinical Review 99. The management of Graves' Disease in Children, with special emphasis on Radioiodine traetment. J Clin Endocrinol Metab 1998; 83: 3767-76.

15.- Nabham Z, Kreher N, Eugster E: Hashitoxicosis in children: clinical features and natural history. J Pediatrics 2005; 146: 533-6.

16.- Zimmerman D, Gan-Paisano M: Hyperthyroidism in children and adolescents. Ped Clin Noth-Am 1990; 37: 1273-89.

17.- McLachlan SM, Nagayama Y, Rapoport B: Insight into Graves' hyperthyroidism from animal models. Endocr Rev 2005; 26: 800-82.

18.- Weetman A: Graves Disease. N Engl J Med 2000; 343 : 1236-48.

19.- Cooper D: Antithyroid drugs. N Engl J Med 2005; 352: 905-17.

20.- de Luis DA, Arconada A, Aller R, Cuellar L, Terrosa $M C$, Martín J: Evolución clínica de una cohorte de pacientes con enfermedad de Graves-Basedow tratados con metimazol. Med Clin 2002; 118: 777-8.

21.- Chih-Tsueng He, An-Tsz Hsieh, Dee Pei, et al: Comparision of single dayly dose of methimazole and propylthiouracil in the treatment of Graves hyperthyroidism. Clin Endocrinol 2004; 60: 676-81.

22.- Hashizume K, Ichikawa I, Sakurai A, et al: Administration of thyroxine in treated Graves' Disease. N Engl J Med 1991; 324: 947-53.

23.- Cooper D: Antithyroid drugs in the management of patients with Graves Disease: an evidence-based approach to therapeutic controversies J Clin Endocrinol Metab 2003; 88: 3474-81.

24.- Michelangeli V, Poon C, Taft J, Newnham H, Topliss $D$, Colman $P$ : The prognostic value of thyrotropin receptor antibody measurement in the early stages of treatment of Graves' disease with antithyroid drugs. Thyroid 1998; 8: 119-24.

25.- Rivkees $S$ : Radioactive iodine use in childhood Graves disease: Time to wake up and smell the I-131 J Clin Endocrinol Metab 2004; 89: 4227-28.

26.- Astl J, Dvorakova M, Vicek P, Vesely D, Matucha P, Betka J: Thyroid surgery in children and adolescents. Internat J Ped Otorh 2004; 68: 1273-78.

27.- Read C Jr, Tansey M, Menda Y: A 36-year retrospective análisis of efficay and safety of radioactive iodine in treating young Graves patients. J Clin Endocrinol Metab 2001; 14: 229-43. 